\title{
Electropalatography for articulation disorders associated with cleft palate (Review)
}

\author{
Lee ASY, Law J, Gibbon FE
}

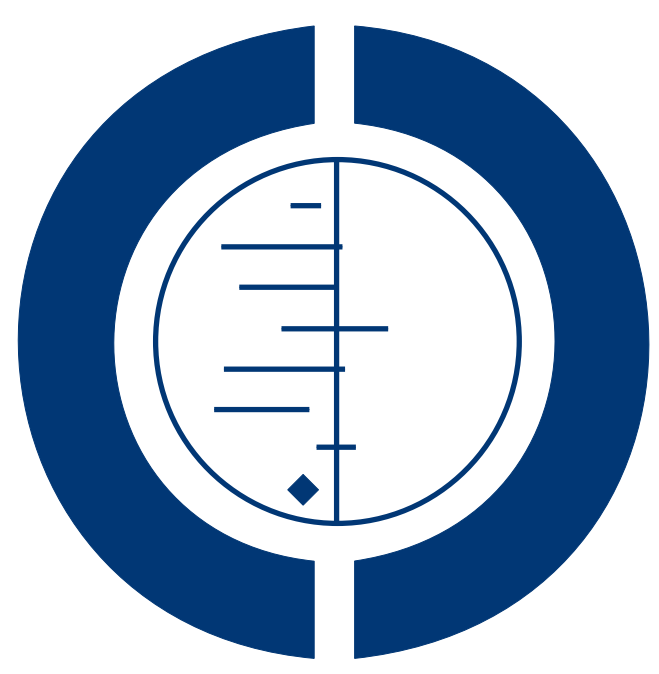

\section{THE COCHRANE COLLABORATION $^{\circledR}$}

This is a reprint of a Cochrane review, prepared and maintained by The Cochrane Collaboration and published in The Cochrane Library 2009, Issue 3

http://www.thecochranelibrary.com

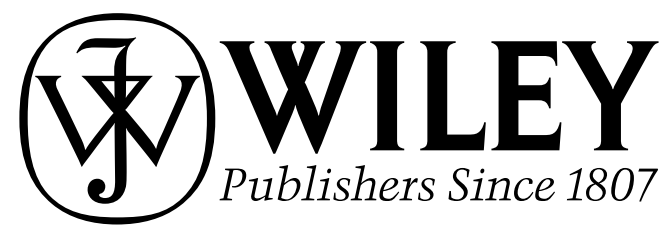

Electropalatography for articulation disorders associated with cleft palate (Review)

Copyright (? 2009 The Cochrane Collaboration. Published by John Wiley \& Sons, Ltd. 
TABLE OF CONTENTS

HEADER . . . . . . . . . . . . . . . . . . . . . . . . . . . . . . . . . . . . . . . . 1

ABSTRACT . . . . . . . . . . . . . . . . . . . . . . . . . . . . . . . . . . . . . . 1

PLAIN LANGUAGE SUMMARY . . . . . . . . . . . . . . . . . . . . . . . . . . . . . . . . . . . . . . $\quad . \quad 2$

BACKGROUND . . . . . . . . . . . . . . . . . . . . . . . . . . . . . . . . . . . . . 2

OBJECTIVES . . . . . . . . . . . . . . . . . . . . . . . . . . . . . . . . . . . . . . . . . . . . . . . 4

METHODS . . . . . . . . . . . . . . . . . . . . . . . . . . . . . . . . . . . . . . . 44

RESULTS . . . . . . . . . . . . . . . . . . . . . . . . . . . . . . . . . . . . . . . 8

Figure 1. . . . . . . . . . . . . . . . . . . . . . . . . . . . . . . . . . . . . . 8

DISCUSSION . . . . . . . . . . . . . . . . . . . . . . . . . . . . . . . . . . . . . $\quad .10$

AUTHORS' CONCLUSIONS . . . . . . . . . . . . . . . . . . . . . . . . . . . . . . . . . . . . . . . . 12

ACKNOWLEDGEMENTS . . . . . . . . . . . . . . . . . . . . . . . . . . . . . . . . . . . . 12

REFERENCES . . . . . . . . . . . . . . . . . . . . . . . . . . . . . . . . . . . . . 13

CHARACTERISTICS OF STUDIES . . . . . . . . . . . . . . . . . . . . . . . . . . . . . . . . . . . . . . 15

DATA AND ANALYSES . . . . . . . . . . . . . . . . . . . . . . . . . . . . . . . . . . . . . . 19

APPENDICES . . . . . . . . . . . . . . . . . . . . . . . . . . . . . . . . . . . . . . . . . . 19

WHAT'S NEW . . . . . . . . . . . . . . . . . . . . . . . . . . . . . . . . . . . . . 21

HISTORY . . . . . . . . . . . . . . . . . . . . . . . . . . . . . . . . . . . . . . . 21

CONTRIBUTIONS OF AUTHORS . . . . . . . . . . . . . . . . . . . . . . . . . . . . . . . . . . . . . 22

DECLARATIONS OF INTEREST . . . . . . . . . . . . . . . . . . . . . . . . . . . . . . . . . . . . 22

DIFFERENCES BETWEEN PROTOCOL AND REVIEW . . . . . . . . . . . . . . . . . . . . . . . . . . 22 


\title{
Electropalatography for articulation disorders associated with cleft palate
}

\author{
Alice S-Y Lee ${ }^{2}$, James Law ${ }^{3}$, Fiona E. Gibbon ${ }^{1}$ \\ ${ }^{1}$ Department of Speech and Hearing Sciences, University College Cork, Cork, UK. ${ }^{2}$ Department of Speech and Hearing Sciences, \\ University College Cork, Cork, Ireland. ${ }^{3}$ Centre for Integrated Healthcare Research, Queen Margaret University, Edinburgh, UK \\ Contact address: Fiona E. Gibbon, Department of Speech and Hearing Sciences, University College Cork, Brookfield Health Sciences \\ Complex, College Road, Cork, UK. f.gibbon@ucc.ie. (Editorial group: Cochrane Developmental, Psychosocial and Learning Problems \\ Group.)
}

Cochrane Database of Systematic Reviews, Issue 3, 2009 (Status in this issue: New)

Copyright (c) 2009 The Cochrane Collaboration. Published by John Wiley \& Sons, Ltd.

DOI: $10.1002 / 14651858$.CD006854.pub2

This version first published online: 8 July 2009 in Issue 3, 2009.

Last assessed as up-to-date: 8 April 2008. (Help document - Dates and Statuses explained)

This record should be cited as: Lee ASY, Law J, Gibbon FE. Electropalatography for articulation disorders associated with cleft palate. Cochrane Database of Systematic Reviews 2009, Issue 3. Art. No.: CD006854. DOI: 10.1002/14651858.CD006854.pub2.

\begin{abstract}
A B S T R A C T
Background

Cleft palate is the most common congenital deformity of the face. It could affect speech acquisition, resulting in articulation errors that could persist into adulthood. Electropalatography (EPG) has been used in speech therapy with individuals who have articulation problems that are unresponsive to "standard treatment" procedures.
\end{abstract}

\section{Objectives}

To determine the effectiveness of speech intervention using electropalatography (EPG) for treating articulation errors in individuals with repaired cleft palate.

\section{Search strategy}

The following databases were searched: CENTRAL 2008 (Issue1), MEDLINE 1966 to March 2008, EMBASE 1974 to March 2008, CINAHL 1982 to March 2008, PsycINFO 1967 to March 2008 and eight other databases. We handsearched Clinical Linguistics and Phonetics (1987 to 2008, Issue 2), Cleft Palate Journal/ Cleft Palate-Craniofacial Journal (1980 to 2008, Issue 1), and the International Journal of Language and Communication Disorders (1980 to 2008, Issue 1). We searched the EPG bibliography (Gibbon 2007). We reviewed reference lists of relevant articles and approached researchers to identify other possible published and unpublished studies.

\section{Selection criteria}

Randomised controlled studies comparing EPG intervention to no treatment, delayed treatment, "standard treatment", or alternative treatment techniques for managing articulation problems associated with cleft palate in children or adults.

\section{Data collection and analysis}

One author searched the titles and abstracts and assessed trial quality. A second author checked judgements; disagreement was resolved through discussion. Three authors were available to examine any potential trials for possible inclusion in the review.

Main results

Electropalatography for articulation disorders associated with cleft palate (Review)

Copyright (c) 2009 The Cochrane Collaboration. Published by John Wiley \& Sons, Ltd. 
One trial using parallel design met the inclusion criteria of this review; no meta-analysis was performed. The study reported that fewer therapy sessions were needed to achieve the treatment goals for the EPG therapy and frication display method $(\mathrm{N}=2)$, followed by EPG therapy $(\mathrm{N}=2)$ and "standard treatment" $(\mathrm{N}=2)$.

\section{Authors' conclusions}

The included trial was a small-scaled study and there were serious limitations in the design and methodology (e.g. allocation concealment was unclear, blinding of outcome assessor(s) was not ensured, few quantitative outcome measures were used, and the results were not reported as planned). Therefore, the current evidence supporting the efficacy of EPG is not strong and there remains a need for highquality randomised controlled trials to be undertaken in this area.

\section{PLAIN LANGUAGE SUMMARY}

\section{Electropalatography for articulation disorders associated with cleft palate}

A cleft palate means that during the early stages of pregnancy, the baby's roof of the mouth does not join in the normal way. The lip is sometimes cleft as well as the palate. Children who are born with a cleft lip or palate usually have corrective surgery during the first few years of life. Nowadays, surgery is so good that there are few long term consequences.

However, some children with repaired cleft palate can have difficulties with speech as they get older. One type of speech difficulty affects consonants formed by the tongue in the mouth, such as $/ \mathrm{t} /, / \mathrm{d} /, \mathrm{s} /$. This makes words like "toe", "door" and "sun" sound distorted or even unrecognisable to listeners. These consonants can be difficult to correct, even by experienced speech and language therapists. Any long term speech difficulties are likely to have a negative effect on children's social, psychological and educational development and future prospects.

One technique that may help to correct these abnormal articulations is electropalatography or EPG. EPG is a computer-based technique that can display the tongue's contact with the hard palate (roof of the mouth) during speech. It involves the child wearing an adapted dental plate, with sensors on the surface. When the tongue contacts the sensors, distinctive patterns for consonants are displayed on a computer screen. The patterns can be used in speech therapy to provide visual feedback so that children can learn normal patterns for consonants they find difficult.

It is not known whether EPG benefits children with cleft palate. Such information is important for speech and language therapists who may want to use the technique in their clinical practice. The review reveals that at present there are no high quality (randomised controlled trial) studies in this field to enable conclusions to be drawn about the efficacy of treatment using EPG for children with cleft palate.

\section{B A C K G ROU N D}

\section{Description of the condition}

Cleft palate is the most common birth defect and the most common congenital deformity of the face (Kummer 2001). Its incidence varies from 1:500 to 1:2000, depending on racial background and gender (Marazita 2004). Cleft palate occurs when the roof of the mouth has not joined completely due to abnormal facial development during the fourth to twelve weeks of the gestation period. The cleft "can range from just an opening at the back of the soft palate to a nearly complete separation of the roof of the mouth (soft and hard palate)" (CLAPA 2001). It may occur on one side (unilateral) or on both sides (bilateral). Cleft palate is usually closed surgically by the age of 18 months (Seagle 2004; Watson 2001). However, the timing of surgical intervention varies between centres (Liao 2006).

\section{Cleft palate and syndromes}

Cleft palate may be associated with other congenital anomalies, and may occur as a part of a well-defined syndrome, such as velocardiofacial syndrome. To date, there are more than 400 syndromes that include cleft palate as a feature (Winter 2000) and this accounts for about $30 \%$ of the cleft cases. Individuals born with cleft palate are more likely to have cognitive deficits than normal children, with an even higher risk in children born with a syndrome (Endriga 1999), possibly due to aberrant brain devel- 
opment (Nopoulos 2002) and other factors. Cognitive problems associated with various types of learning disabilities can add to the detrimental effect of palatal cleft on speech acquisition.

\section{Compensatory misarticulations}

The impact of cleft palate on speech acquisition is apparent early, at the stage of pre-speech vocalisations, and its influence may continue even after surgical intervention (Peterson 2006). It has been estimated that normal articulation can be expected in $25 \%$ of preschoolers with repaired cleft palate who receive routine care by a cleft palate team, but "a significant number of individuals will continue to demonstrate problems with articulation in adolescence" (Peterson 2001). Compensatory misarticulations are one of the prominent articulation problems observed in individuals with a history of cleft palate. The prevalence of compensatory misarticulations in English-speaking children born with cleft palate varies from $22 \%$ to $28 \%$ (Dalston 1992; Hardin-Jones 2005; Peterson 1990 ) and a recent study reported a prevalence of $28.5 \%$ in Greekspeaking children (Paliobei 2005). Compensatory misarticulations are learned articulation deviations and most of them are errors in place of articulation (Peterson 2006). For example, the sound $/ \mathrm{t} /$ is backed from the alveolar region to the velar region, hence, listeners perceived a $/ \mathrm{k} /$ sound. They are believed to result from strategies developed by individuals with cleft palate as a response to inadequate intraoral pressure for normal articulation caused by the structural deficit (Warren 1990). Once learned, these abnormal learned patterns can persist due to habituation (Peterson 2001). Since compensatory misarticulations are due to abnormal learning, they are under the speaker's control and, therefore, can respond to speech therapy (Kummer 2001).

\section{Description of the intervention}

\section{Speech therapy}

It is estimated that between $50-75 \%$ of individuals born with cleft palate require speech intervention at some point in their lives ( Peterson 2001; Witzel 1991). Based on a comprehensive speech assessment, speech-language therapists (SLTs) apply phonetic and phonological approaches and principles of behaviour modification in speech therapy (Sell 2001). The SLTs teach directly the target speech sounds to the individuals, with the aid of different techniques and tools such as tactile cues, the use of mirror, and diagram of the oral cavity. Once the target production is established, the sound is practised and stabilised in a hierarchical progression of speech contexts, from syllables through words, phrases, sentences to spontaneous speech (Peterson 2006). The SLTs also work with the individuals on their speech sound discrimination and self-monitoring skills in order to establish internal mechanisms for correct target selection and perceptual-motor self-monitoring
(Sell 2001). However, not all individuals with compensatory errors respond well to this "standard treatment" approach; posterior articulation pattern persists in some individuals despite of years of speech therapy (Sell 2001). Especially in adolescents and adults, modification is more likely to occur if some type of biofeedback therapy, such as speech therapy using electropalatography (EPG), is provided.

\section{Using EPG for speech assessment and therapy}

EPG is a computer-based instrument which gives information on the location and timing of the tongue's contact with the hard palate during speech (Hardcastle 1991; Hardcastle 1997). It has been used to assess lingual articulation and to treat articulatory disorders through the use of visual feedback (a type of biofeedback) during speech therapy. Each patient undergoing EPG assessment or therapy has to wear a custom-made artificial dental plate which is moulded to fit the speaker's hard palate. The dental plate is embedded with electrodes on the lingual surface. When the electrodes are contacted by the tongue during speech, a signal is sent to an external processing unit through lead-out wires and real-time visual feedback of the location and time of tongue-palate contacts is shown on a computer monitor. The SLTs use this visual display to teach their clients to establish new, appropriate articulatory pattern for the speech sounds targeted for treatment.

\section{How the intervention might work}

The use of biofeedback in speech therapy is a clinical application of cybernetic theory, which views behavioural and physiological activity and learning as self-regulated processes that involve specific organically based control mechanisms (Davis 1980, Smith 1967, Wiener 1948). Speech production has been described as a selfregulating, closed-loop or servo- system (Fairbanks 1954, Mysak 1959). The system contains a "comparator" where the input signal and feedback signals of the output (i.e.e.g. auditory, tactile, and proprioceptive feedback) are compared; the presence of difference between them indicates the occurrence of errors. Appropriate information is then sent to a "mixer" which combines the error signal and input signal to alter the operation of the "effectors" (i.e. the anatomical structures involved in respiration, phonation, resonance and articulation; Fairbanks 1954, Mysak 1959). Interruption of the speech control mechanism may cause disturbances of speech production, resulting in, for example, articulation errors. Speech problems can be managed by using biofeedback in speech therapy. Biofeedback involves the use of instrumentation (e.g. EPG) to give explicit, real-time information about a specific physiologic system (e.g. tongue-palate contact) that is under the control of the nervous system but not clearly or accurately perceived by the individual (Davis 1980). The individual can then make use of the information to facilitate the control of the response (e.g. articulation). Biofeedback has been employed in managing 
various communication problems, such as voice disorders and fluency disorders (Davis 1980).

\section{Why it is important to do this review}

The real-time visual feedback provided by EPG has been used in speech therapy with individuals who have abnormal articulations that are unresponsive to "standard treatment" procedures ( Stengelhofen 1990). Although there exists substantial literature about speech intervention using EPG, there has not been a systematic review of the effectiveness of EPG, hence, it remains unknown whether EPG is beneficial and as a result it is difficult for SLTs to make decisions about whether or not to use EPG in their clinical practice. Therefore, this review aimed to assess the effectiveness of EPG in speech therapy for treating articulation errors for individuals with articulation disorders related to cleft palate.

\section{O B J E C T I V E S}

To assess the effectiveness of electropalatography (EPG) in improving the treatment of articulation errors in individuals with repaired cleft palate.

\section{METHODS}

\section{Criteria for considering studies for this review}

\section{Types of studies}

Randomised controlled trials in which participants have been randomly allocated to different treatment conditions by, for example, using a random number table.

\section{Types of participants}

Individuals with articulation problems associated with cleft palate. Included were individuals with recognized syndromes (e.g. velocardiofacial syndrome, Treacher Collins syndromes, etc). There was no restriction on age range.

Those with learning disability associated with severely limited expressive/spoken language (as judged by SLTs using informal or standardised language test) or severe hearing impairment were excluded from the review purely on the assumption that a certain level of cognitive abilities of the individuals is required in order for them to benefit from EPG therapy.

\section{Types of interventions}

Interventions using EPG compared to no treatment, delayed treatment, "standard treatment", or alternative treatment techniques (e.g. treatment using ultrasound).

There was no restriction on the frequency, intensity and duration of speech intervention.

\section{Types of outcome measures}

\section{Primary outcomes}

The primary outcome is the measure of correct articulation of speech sounds targeted in therapy, which could be assessed by:

- Standardised articulation tests (e.g. Edinburgh Articulation Test (Anthony 1971) );

- Other perceptual evaluation of articulation (e.g. Cleft Audit Protocol for Speech, percentage of consonants correct);

- Articulatory accuracy based on instrumental measures, such as electropalatography (EPG);

- Measures of speech intelligibility (e.g. by means of a perceptual rating scale, or by calculating the number of words correctly transcribed by examiner).

\section{Secondary outcomes}

- Measures of listener acceptability (e.g. by using a perceptual rating scale);

- Participants' perceptions of impact of wearing the EPG plate on (i) speech production, (ii) tongue movement, (iii) sensation in the mouth, (iv) self-perceived appearance, (v) gagging, and (vi) saliva;

- Adverse effects.

Outcome measures of interest did not form part of the inclusion criteria.

\section{Search methods for identification of studies}

\section{Electronic searches}

We searched the following databases:

- The Cochrane Central Register of Controlled Trials (The Cochrane Library Issue 1, 2008)

- MEDLINE (1966 to March 2008)

- EMBASE (1974 to March 2008)

- ERIC (1966 to March 2008)

- PsycINFO (1967 to March 2008)

- CINAHL (1982 to March 2008)

- Linguistics and Language Behaviour Abstracts (LLBA) (1973 to March 2008) 
- Allied and Complementary Medicine (AMED) (1985 to March 2008)

- Latin American Health Sciences Literature (LILACS) (1982 to March 2008)

- Japana Centra Revuo Medicina (1983 to February 2008)

- The National Research Register (searched March 2008)

- ClinicalTrials.gov (searched March 2008)

- Dissertation Abstracts (1861 to March 2008)

The following search terms were used to identify articles. No language or date restriction was applied. RCT filters were not used as it was felt that they would restrict the search leading to potentially useful records possibly being missed.

1. cleft palate

2. electropalatograph*

3. EPG

4. palatograph*

5. palatomet*

6. 2 or 3 or 4 or 5

7. 1 and 6

The search stratagies for the individual databases can be found in Appendix 1, Appendix 2, Appendix 3, Appendix 4, Appendix 5, Appendix 6, Appendix 7, Appendix 8, Appendix 9, Appendix 10, Appendix 11, and Appendix 12.

\section{Searching other resources}

We handsearched three journals from 1980 onwards - Clinical Linguistics and Phonetics (1987 to 2008, Issue 2), Cleft Palate Journall Cleft Palate-Craniofacial Journal (1980 to 2008, Issue 1), and the International Journal of Language and Communication Disorders (1980 to 2008, Issue 1). The Master List of Journals (http://www.cochrane.us/masterlist.asp) was checked to ensure that they had not been handsearched by The Cochrane Collaboration. We searched the EPG bibliography (Gibbon 2007). We reviewed reference lists of relevant articles and approached colleagues and researchers to identify other possible published and unpublished studies such as technical or research reports, conference papers, and different types of dissertations. There was no language restriction; no translation was sought since no non-English potential study was identified.

\section{Data collection and analysis}

\section{Selection of studies}

$\mathrm{AL}$ conducted the electronic search and FG was in charge of the handsearch. All references generated from the search strategy were managed using a reference management programme. AL independently examined the studies against the inclusion criteria. The judgements were then checked by FG and any disagreement was resolved through discussion. Full text version was obtained for articles that appeared to have met the inclusion criteria or those that seemed to be uncertain if they have met the inclusion criteria. Articles that did not fulfil the inclusion criteria were discarded. The reviewers were not blinded to the name(s) of the author(s), institution(s) or publication source at any level of review.

\section{Data extraction and management}

A paper data extraction form was developed for extracting the following information:

\section{Participants}

1. Number of participants

2. Age

3. Sex

4. Inclusion and/or exclusion criteria

5. Level of severity of articulation disorders

6. Reason(s) for patients being rejected, if applicable

7. Other baseline characteristics of the participants reported e.g. hearing ability

\section{Methods}

1. Type(s) of speech assessment conducted

2. Assessment findings (e.g. number and types of articulation errors, such as double articulation, palatalization)

\section{Interventions}

1. Type of interventions

2. Length of intervention i.e. number of hours of the course of speech therapy

3. Number of therapy session

4. Whether compliance evaluated

\section{Outcomes}

1. Total numbers and events for each intervention group or risk ratio and its error (binary outcomes)

2. Mean and deviation (continuous data)

\section{Assessment of risk of bias in included studies}

AL independently assessed the risk of bias in each included study under the following six domains. FG checked and agreed with the judgements. JL was available for consultation in case there was disagreement between AL and FG and a consensus between them could not be reached.

The assessment of risk of bias consisted two parts: (1) a succinct description, which included verbatim quotes from reports or correspondence and/or a comment from the reviewer, of the procedures conducted to avoid bias; and (2) a judgement of the risk of bias based on part 1. A judgement of 'Yes' means that there was low risk of bias in the study; whereas 'No' indicates a high risk 
of bias in the included study (see also the Cochrane Handbook, Higgins 2008). A judgement of 'Unclear' means that the risk of bias was uncertain due to insufficient information available for the judgement. The judgement criteria for each domain are described below.

\section{Sequence generation}

The method used to generate the allocation sequence was described using quotes wherever possible. Comment, such as "probably done" or "probably not done", was added to supplement any ambiguous quote. Each included study was assigned to one of the following categories:

- 'Yes' indicates adequate method was used for randomisation e.g. using computer generated or table of random numbers.

- 'Unclear' indicates uncertainty about whether appropriate method of randomisation was used.

- 'No' indicates that inadequate method of randomisation was used e.g. case file number, date of birth, or alternate numbers.

\section{Allocation concealment}

The included studies was assigned to one of the following quality criteria whereby:

- 'Yes' indicates adequate concealment of allocation e.g. prenumbered or coded identical containers administered serially to participants.

- 'Unclear' indicates uncertainty about whether the allocation was adequately concealed e.g. the authors did not describe the allocation methods.

- 'No' indicates that the allocation was not adequately concealed e.g. alternate assignment.

For those studies assessed as 'Unclear' or 'No', the pre-treatment assessment and the allocation of participants was described in the review (Description of Studies) in order to identify differences between intervention and control groups that can be ascertained at baseline.

\section{Blinding}

Blinding of participants and healthcare providers (i.e. the SLTs) is not possible for EPG therapy but blinding of outcome assessor(s) and data analyst(s) from knowledge of which intervention a participant had received should be ensured. The measures used to ensure blinding were described and evaluated. This domain was graded as 'Yes', 'Unclear', or 'No'. Assessment was made for each main outcome (e.g. outcome measured at 6 months post-therapy, outcome measured at 12 months post-therapy).
Incomplete outcome data refers to those that were due to attrition (drop-out) during the study, or exclusions from the analysis. Protection from attrition bias includes:

- Attrition described, proportion smaller than $10 \%$ of assigned patients and difference between the two groups not greater than $10 \%$.

- Reason(s) of participants withdrawal explained.

- Attrition appropriately analysed (e.g. intention-to-treat analysis).

\section{Selective reporting bias}

This is also known as within-study publication bias. It may arise in several ways (Higgins 2008):

- Only some of the analysed outcomes were included in the study.

- Different time points at which the outcome was measured, or different instruments or assessors employed to measure the outcome at the same time point.

- Selective reporting of analysis using the same data.

- Selective reporting the results of sub-scales of full measurement scale or a subset of events.

- Some outcomes were reported but with inadequate detail for the data to be included in a meta-analysis.

\section{Other sources of bias}

Other possible sources of bias include:

- Baseline imbalance - measures used to ensure the groups compared in the study were similar at baseline in terms of severity of articulation problems and other baseline characteristics (e.g. hearing ability) would be described.

- Early stopping - incidence of early stopping of intervention and the reason(s) for stopping early would be described.

- Co-intervention - whether some of the participants received additional intervention (e.g. speech therapy) would be described.

\section{Measures of treatment effect}

Further analysis was not carried out as only one trial was identified. Methods planned in the protocol are included below and they will be used in future updates.

\section{Binary data}

Binary data may be likely (e.g. "articulation improved versus no change"). The data will be analysed by calculating the risk ratio. 
Standardised articulation test results, articulation accuracy based on EPG or perceptual evaluation, judgement of speech intelligibility and listener acceptability will be treated as continuous data. Weighted mean difference (WMD, or the 'difference in means') will be calculated if the outcome measurements in all studies are made on the same scale. Otherwise, standardised mean differences (SMD) will be used to combine studies that measured the same outcome using different methods.

\section{Unit of analysis issues}

\section{Cluster-randomised trials}

Clustering effects should be taken into account by analysing the data using appropriate statistical approaches (e.g. using two-sample $\mathrm{t}$-test to compare the means of the clusters in the intervention group to those in the control group at cluster-level; or mixed effects linear regression approach at individual-level; Donner 2000). The author(s) will be contacted in case this was not described clearly in the study. If appropriate controls of clustering were not used, individual participant data will be requested and re-analysed using multilevel models (Donner 2000). The results will be combined with those from individually randomised trials for meta-analysis if the clinical heterogeneity between studies is small (Donner 2001).

\section{Cross-over trials}

Cross-over trials are not appropriate for intervention that can have a lasting effect (Higgins 2008). Therefore, this design is not suitable for studying interventions for treating articulation problems.

\section{Studies with multiple treatment groups}

Since three comparisons were planned (see Data analysis below), the "shared" group (i.e. the group that received EPG therapy) will be split into two or more groups of smaller sample size for independent comparisons (Higgins 2008).

\section{Dealing with missing data}

Authors will be contacted and asked to supply missing data. In the event that the authors could not be contacted or data could not be supplied, missing data and drop-outs will be assessed for each included study and the number of participants who were included in the final analysis as a proportion of all participants in each study will be reported. Where known, reasons for missing data will be provided.

\section{Assessment of heterogeneity}

Clinical heterogeneity will be assessed by examining the types of participants, the severity of articulation errors, interventions, and outcome measures as specified in the criteria for included studies. The consistency across studies will be assessed using the Chi- squared test for heterogeneity, through visual inspection of forest plots, and by using $\mathrm{I}^{2}$ test (Higgins 2002, Higgins 2003). $\mathrm{I}^{2}$ is a quantity that describes the percentage of variability in point estimates that is due to heterogeneity rather than sampling error. If there is evidence of heterogeneity (i.e. an $\mathrm{I}^{2}$ value of $25 \%$ or greater), the possible causes of heterogeneity will be explored by examining the studies and data again and where appropriate conducting subgroup analyses.

\section{Assessment of reporting biases}

Funnel plots (effect size against error) will be drawn if sufficient studies were found. An asymmetric funnel indicates a relationship between effect size and study size, which suggests the possibility of either publication bias or a systematic difference between smaller and larger studies. If a relationship was identified, the clinical diversity of the studies will also be examined (Egger 1997).

\section{Data synthesis}

\section{Data analysis}

The following comparisons will be made:

1. EPG therapy versus delayed or no treatment

2. EPG therapy versus "standard treatment"

3. EPG therapy versus alternative treatment technique

\section{Meta-analysis}

Meta-analysis will be carried out using the RevMan 5.0 statistical package, if there are sufficient data. A fixed effects model will be used for analysis. If there is statistical heterogeneity which cannot be explained (e.g. by means of subgroups), a random effects metaanalysis will be used to incorporate heterogeneity among studies. A random effects meta-analysis model assumes that the effects being estimated in the different studies are not identical but follow some common distribution.

\section{Subgroup analysis and investigation of heterogeneity}

If possible, subgroup analyses will be conducted to assess the impact of individual's age and intensity of therapy (number and frequency of speech therapy sessions). Interpretation of the findings from multiple subgroup analyses will consider the possibility of false positive findings (Higgins 2005).

\section{Sensitivity analysis}

Sensitivity analyses will be performed to assess the robustness of conclusions by examining the impact of study quality. The factors that are considered as important to study quality include randomisation, blinding to outcome assessment, and attrition (Juni 2001). Studies which are categorised as (A) or (B) for these factors in the methodological quality assessment will be included in the analysis. 


\section{R E S U L T S}

\section{Description of studies}

See: Characteristics of included studies; Characteristics of excluded studies.

See also Figure 1 for a summary of the results of the search and the Characteristics of included studies and Characteristics of excluded studies tables.

Figure I. Tree diagram summarising the results of the literature search.

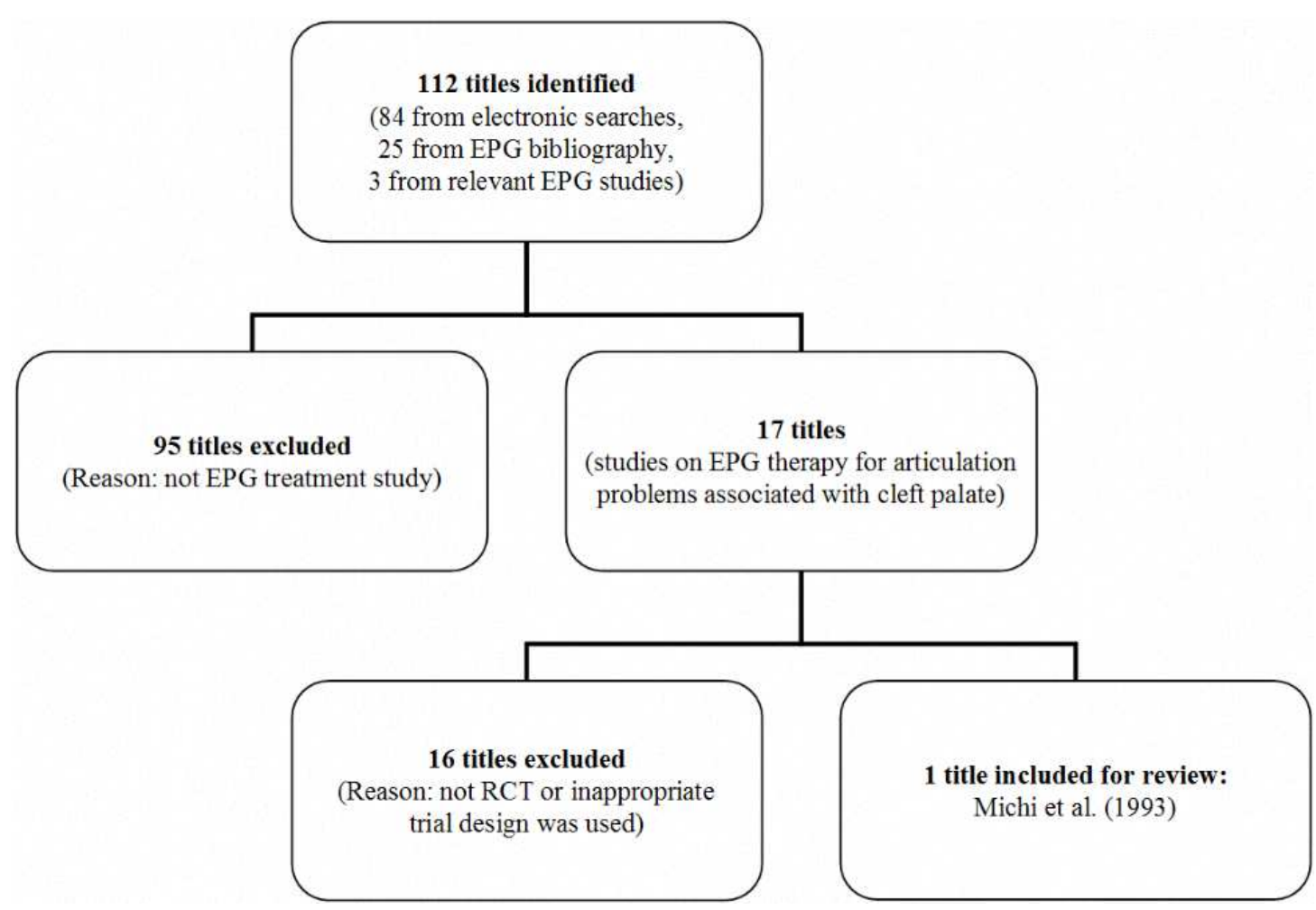

\section{Results of the search}

The literature review identified 112 titles - 84 titles through electronic searches, 25 from the EPG bibliography (Gibbon 2007), and 3 were identified from relevant EPG studies (Michi 1986, Michi 1993, Yamashita 1992). The studies were examined and two were initially judged to have met the inclusion criteria: a study by Gibbon and colleagues (Gibbon 2001) and a study by Michi and associates (Michi 1993). However, the Gibbon et al. study was excluded because cross-over design, which is considered as inappropriate for studying speech intervention, was used. Thus, the Michi et al. study is the only trial included in this review.

\section{Included studies}

\section{Participants}

All participants $(\mathrm{N}=6)$ in Michi et al.'s study were Japanesespeaking children, aged 4-6 years (Michi 1993). None of them has any significant additional impairment, such as mental disability or hearing impairment. All participants had compensatory misarticulations associated with cleft palate.

\section{Interventions}

Michi et al. used parallel group design and compared three treatment conditions: (1) EPG and friction display method, (2) EPG therapy and (3) no-visual-feedback method. The participants received a 60-minute individual therapy session once per week, except for one participant who was given daily treatment in order to 
accommodate his schedule (Michi 1993). Michi et al. used the realtime EPG contact pattern display as a visual feedback to aid the teaching of the appropriate tongue placement for speech sound $/ \mathrm{s} /$. There were four stages in the treatment, where the initial stage was to establish the production of an intermediate simplified pattern and the final stage aimed at the production of a nearly normal pattern using appropriate direction and amount of air stream. Speech stimuli of different linguistic complexity were gone through at each stage in a hierarchy from isolated sound, through syllable to word level (Michi 1993). The EPG and frication display method included EPG therapy and the use of waveform models of frication produced by a speech-language pathologist as visual feedback for treating frication production. The no-visual-feedback method did not involve the use of any additional technique or equipment in the therapy and therefore it is considered as "standard treatment".

\section{Outcome measures}

The outcome measures included: (1) percent correct tongue placement, as judged by examining the EPG patterns, and (2) percent correct production of frication, as determined by perceptual judgement (Michi 1993). A baseline over one month was established and the progress was assessed at the onset and end of each therapy session (Michi 1993).

\section{Excluded studies}

Most of the titles found through literature search were not treatment studies - they were studies that investigated the tongue-palate contact patterns in normal articulations, or in articulation errors associated with cleft palate with comparison to some normative EPG data. Seventeen of the titles were on EPG treatment for articulation problems associated with cleft palate. These potentially relevant studies were examined but they were excluded from this review because one used cross-over design (Gibbon 2001), which is inappropriate for evaluating speech intervention, and the other 15 titles are not randomised controlled trials: nine were single case study (Dent 1992, Gibbon 1989, Gibbon 1998, Hardcastle 1989, Michi 1985, Michi 1986, Ohira 1989, Scobbie 2004, Whitehill 1996); four were case study series (Fujiwara 2007, Schmidt 2007, Stokes 1996, Yamashita 1988); and two were before and after study, with control group (Michi 1990, Yamashita 1999).

\section{Risk of bias in included studies}

See also the Risk of bias table of the included studies.

\section{Allocation}

Michi et al. stated explicitly that randomisation was used to assign participants to different treatment conditions, however, the method of randomisation was not specified in the study (Michi 1993). Hence, adequate allocation concealment is unclear.

\section{Blinding}

Michi et al. did not state whether the second author was blinded to treatment allocation at the time of outcome assessment (Michi 1993).

\section{Follow up and exclusions}

No drop-out was reported in the study (Michi 1993).

\section{Selective reporting}

Michi et al. did not report all results of the treatment outcomes as planned. The authors mentioned the measure of percent correct production for tongue placement and frication for assessing the treatment progress in the Method section. However, they reported the number of therapy sessions each participant required to achieve the targets for some of the stages of the interventions (Michi 1993). Some charts were included to provide additional information on this but they were difficult to interpret.

\section{Other potential sources of bias}

All participants showed the same type of articulation errors before the intervention began (Michi 1993). No early stopping of intervention and co-intervention were reported.

\section{Effects of interventions}

Meta-analysis was not performed as only one study was included in this review. The Michi et al. study used a descriptive method to assess the treatment outcome and no statistical analysis was conducted (Michi 1993).

\section{Primary outcomes}

\section{Standardised articulation tests}

Michi et al. did not use any standardised articulation test to measure the treatment outcome.

\section{Other perceptual evaluation of articulation}

\section{Percent correct production}

Michi et al. measured the percent correct production for tongue placement based on visual inspection of EPG patterns and frication based on perceptual judgement made by the second author of the study at the start and end of each therapy session (Michi 1993). A criterion of at least $80 \%$ accuracy was used for determining whether the treatment should progress to the next level or not. However, in the Results section, the authors reported mainly the number of therapy sessions each participant required to achieve the treatment target for some of the stages of the interventions. See Additional Table 1 for details. 
Table 1. A summary of the number of therapy sessions required for each stage of the interventions included in the study by Michi et al. (1993). "a" means that the information was not reported and "b" means that the target was attained and the treatment progressed to the next stage within the same session.

\begin{tabular}{|c|c|c|c|c|c|}
\hline Intervention & Subject & Stage I & Stage II & Stage III & Stage IV \\
\hline \multirow{2}{*}{$\begin{array}{l}\text { EPG therapy } \\
\text { and frication display } \\
\text { method }\end{array}$} & 1 & 3 & 1 & a & a \\
\hline & 4 & 3 & 5 & a & a \\
\hline \multirow[t]{2}{*}{ EPG therapy } & 2 & 11 & a & a & a \\
\hline & 5 & $\mathrm{~b}$ & $\mathrm{~b}$ & 5 & 2 \\
\hline \multirow{2}{*}{$\begin{array}{l}\text { No-visual-feedback } \\
\text { method/“standard } \\
\text { treatment" }\end{array}$} & 3 & $\mathrm{~b}$ & $\mathrm{~b}$ & 4 & 6 \\
\hline & 6 & $\mathrm{~b}$ & $\mathrm{~b}$ & 5 & 7 \\
\hline
\end{tabular}

\section{Articulatory accuracy based on EPG}

Michi et al. did not use any of these measures to assess treatment outcome.

\section{Measures of speech intelligibility}

Michi et al. did not measure speech intelligibility to assess treatment outcome.

\section{Secondary outcomes}

Michi et al. did not measure listener acceptability, participants' perceptions of impact of wearing the EPG plate and adverse effects in their study.

\section{DISCUSSION}

Individuals with a history of cleft palate are likely to exhibit articulation problems, which could hamper communication and impair self-esteem. Articulation problems due to abnormal learning do not resolve spontaneously after surgery and they could be resistant to speech intervention as well. It could be frustrating for these individuals and their family members to go through years of therapy and achieve only a small progress. Alternate intervention approach that could manage these persistent articulation problems more effectively and reduce the therapy time is of immense importance for improving the service delivery, which would in turn benefit the various stakeholders in healthcare. This review aimed to address this issue by evaluating the effectiveness of electropalatography (EPG) in treating articulation disorders associated with cleft palate.

\section{Summary of main results}

A comprehensive search of literature revealed a number of EPG intervention studies for cleft palate. However, most of them employed either single-case or small-N research design. Only one randomised controlled trial met the inclusion criteria of the present review (Michi 1993) and, therefore, meta-analysis was not performed. The Michi et al. study used a descriptive approach to evaluate the treatment outcome and no statistical analysis was conducted. The authors reported that less therapy sessions were needed to attain treatment goals for the EPG and frication display method, followed by the EPG method and no-visual-feedback method/"standard treatment" (Michi 1993). There was no report of any harm or adverse effect brought by the EPG therapy in the study.

\section{Overall completeness and applicability of evidence}

The Michi et al. study included only children with articulation errors associated with cleft palate as subjects (Michi 1993). This is clinically relevant to a certain extent as SLTs usually have more paediatric clients than adult clients in their caseload but the evi- 
dence is not complete, as the findings for children may not be generalisable to adult clients. Moreover, the study had not included individuals with recognized syndromes, such as velocardiofacial syndrome, hence, it is uncertain if EPG therapy would benefit this group of clients.

Relevant interventions for treating articulation problems associated with cleft palate have been investigated in the included trial. In clinical practice, "standard treatment" is the conventional treatment approach; EPG therapy may be given to clients who show persistent articulation errors if the clinic is equipped with an EPG machine, the clinician has been trained in using EPG for treatment and resources are available for making an EPG plate for the clients.

For the types of outcome measures, the included trial (Michi 1993) used limited number of assessment methods and the measures are based on EPG (except perceptual judgement of frication produced). Additional parameters, such as standardised articulation tests, speech assessment protocol specific for cleft palate (e.g. Cleft Audit Protocol for Speech used in the UK), measures of speech intelligibility and listener acceptability, were not included. These parameters are important, for example, standardised tests are often used for evaluating the outcome of speech therapy and surgical treatment for cleft palate management; perceptual judgement of speech intelligibility and listener acceptability could provide information on the change in overall speech production as a result of the change in tongue-palate contact patterns. Hence, they should have been included for evaluating treatment outcome.

\section{Quality of the evidence}

There were a number of limitations in the methodology of the included study (Michi 1993). First, the details of allocation concealment were not provided and blinding of outcome assessor(s) was not employed. As stated above, few quantitative outcome measures were used to show the effect of treatment on improving articulation or speech intelligibility. In addition, the results of the outcome measure were not reported as planned. Furthermore, generalisation and maintenance of treatment effect were not assessed. The sample size was very small and there was no attempt to calculate the size of sample required before subject recruitment. Considering these serious limitations in methodology, the level of quality of the evidence provided by the included trials is judged to be very low (Higgins 2008).

\section{Potential biases in the review process}

Every attempt has been made to identify studies that meet the inclusion criteria.

\section{Agreements and disagreements with other}

\section{studies or reviews}

Other EPG treatment studies are the 16 titles that were being excluded from this review - 15 case reports and one cross-over trial. No systematic review on speech therapy using EPG has been conducted previously. There are two brief overviews of EPG as a clinical tool: (1) the NICE (National Institute for Clinical Excellence) interventional procedures overview of electropalatography, published in 2002 (NICE 2002), and (2) the Royal College of Speech and Language Therapists Clinical Guidelines (RCSLT 2005).

The main findings of the included study was that fewer therapy sessions were needed to achieve the treatment goals when EPG was used in therapy, as compared to "standard treatment" (Michi 1993). This is in congruence to the finding of two case studies conducted by Yamashita and colleagues (Yamashita 1988; Yamashita 1999). Other studies also commented that rapid improvement in articulatory patterns was observed during EPG therapy (Fujiwara 2007; Michi 1986; Stokes 1996; Whitehill 1996).

The included study had not investigated the efficacy of EPG in terms of generalization but two case studies had done so and they reported generalization of articulatory pattern from treated targets to untreated speech sounds (Stokes 1996; Whitehill 1996). However, generalization of newly-learned articulatory patterns into everyday speech during the course of EPG therapy was found to be poor for most individuals according to a case study (Michi 1990) and a survey on the SLTs' views on EPG therapy outcomes (Gibbon 2006).

The possibility of adverse effect that EPG may have on individuals undertaking this intervention had not been explored in the included study and other previous EPG treatment studies. However, the NICE interventional procedures overview of EPG commented that this "technique is non-invasive and is unlikely to have severe adverse effects" (NICE 2002).

Overall, the included study and the excluded 16 studies were in the same view that EPG is a useful technique for treating persistent articulation errors. The Michi et al. study and the 15 case studies reported 'success' using EPG therapy. The cross-over study reported 'success' in most of the participants - nine out of 12 showed positive change in the articulatory patterns of the target sounds and three showed no change after the EPG therapy (Gibbon 2001). While for "standard treatment", one participant showed positive change post-intervention, one showed negative change and the rest did not respond to this intervention (Gibbon 2001). This is supported by the SLTs' own clinical experience that the majority of children and adults who received EPG therapy showed at least some success in improving their articulation $(87.5 \% ; \mathrm{N}=21)$ and a small number of individuals $(12.5 \% ; \mathrm{N}=3)$ showed no change in their articulation following the intervention (Gibbon 2006). Clinicians who had used EPG in speech therapy also indicated that the technique had improved their clients' awareness of their articulation difficulties (Gibbon 2006; Stokes 1996; Whitehill 1996) motivation for therapy (Michi 1993; Whitehill 1996). Finally, both NICE interventional procedures overview and RC- 
SLT Clinical Guidelines concluded that there was limited, lowlevel evidence regarding the efficacy of EPG therapy (NICE 2002; RCSLT 2005). The RCSLT Clinical Guidelines recommended further research on the efficacy of EPG therapy. Meanwhile, they suggested "electropalatography should be a treatment option for school-aged children and older patients with persistent articulatory disorders" (RCSLT 2005).

\section{AUTHORS, CONCLUSIONS}

\section{Implications for practice}

To date, the best evidence for the effectiveness of EPG therapy for treating articulation problems associated with cleft palate was one randomised controlled study (Michi 1993). However, the study was of small-scale and there were a number of limitations in the methodology. Despite the recommendation made by the RCSLT, the evidence supporting the efficacy of EPG is not strong and does not support the widespread use of EPG for treating articulation disorders associated with cleft palate. It is recommended that appropriately designed randomised controlled trials should be undertaken before this technique is introduced as part of the routine care of patients with repaired cleft palate.

\section{Implications for research}

Similar to the situation reported in other areas of communication disorders, such as dysarthria (Sellars 2005), the lack of large-scale RCTs in this field is probably because of a combination of factors including the diversity of the problems, patient compliance, and a lack of adequate funding. In addition, there are issues that are pertinent to EPG, such as the time and cost needed for custommaking an EPG plate for each patient, relatively small number of suitable candidates for EPG therapy in each individual cleft palate centre, and the training and support that are needed for the SLTs to use EPG for articulation assessment and treatment.

Although it appears that conducting a large-scale treatment study on EPG therapy is difficult due to the obstacles mentioned above, it is not entirely impossible. In terms of technical issues related to EPG, there is a recent advancement in the technology of making EPG plates, which allows the plates to be custom-made in a much shorter period of time and at a relatively lower cost (Wrench 2007). Regarding subject recruitment issues, a more centralised cleft care service may make the process easier and collaboration between cleft palate centres feasible. For example, in the UK, there has been a recent nationwide reorganisation of cleft services in England, Wales, and Northern Ireland, in response to the concerns on cleft care services and the subsequent review conducted by the research team of the CSAG (Clinical Standards Advisory Group) Cleft Lip and Palate Committee (CSAG 1998). The expertise and resources for cleft care were centralised, resulting in the reduction of $57 \mathrm{cleft}$ units to 12 regional centres throughout the country. Furthermore, the conduction of larger scale treatment study could be facilitated if the infrastructures required are already in place. For example, in the UK, most of the cleft palate centres are now equipped with an EPG system and their SLTs had undertaken training on using EPG for assessment and treatment provided by EPG expert researchers based at Queen Margaret University (QMU) under the CLEFTNET project (Lee 2007). The SLTs had also been given technical support and expert advice on EPG therapy by the QMU research team. To sum up, these recent developments may help the conduction of large-scale RCTs on EPG intervention in the future.

Finally, there are a few recommendations for the planning of EPG treatment studies in the future. The framework for development and evaluation of RCTs for complex interventions proposed by the UK Medical Research Council (MRC 2000) could be applied. This framework consists five different phases: (1) pre-clinical or theoretical, (2) Phase I or modelling, (3) Phase II or exploratory trial, (4) Phase II or main RCT, and (5) Phase IV or long-term surveillance (MRC 2000). According to this model, the findings of the included study (Michi 1993), the results reported in previous case studies and survey (Gibbon 2006), and expert opinions ( Lee 2007) could form the base of the pre-clinical/theoretical phase for developing an RCT (MRC 2000). Further research is needed at this point to identify other "active ingredients" for successful interventions, such as frequency of EPG therapy, clinician characteristics (e.g. experience in EPG therapy), variables related to the individuals receiving the therapy (e.g. cognitive ability) and so on, before embarking on an RCT. Once the efficacy of EPG therapy is established, the focus of research could turn to the development of a treatment paradigm for enhancing generalisation and maintenance of treatment effect. Next, cost-effectiveness analysis, customer satisfaction survey, and quality of life assessment can be carried out in order to appraise the value of EPG therapy (Robey 1998).

\section{ACKNOWLEDGEMENTS}

The reviewers would like to thank Yuri Fujiwara for accessing the Japanese database (Japana Centra Revuo Medicina) and Natalia Zharkova's assistance in handsearching the journals. They also wish to acknowledge Jane Dennis, Jo Abbott and Geraldine Macdonald for their support, and the reviewers for their invaluable comments. 


\section{R E F E R E N C E S}

\section{References to studies included in this review}

\section{Michi 1993 \{published data only\}}

Michi K-I, Yamashita Y, Imai S, Suzuki N, Yoshida H. Role of visua feedback treatment for defective /s/ sounds in patients with cleft palate. Journal of Speech and Hearing Research 1993;36:277-285.

\section{References to studies excluded from this review}

\section{Dent 1992 \{published data only\}}

Dent H, Gibbon F, Hardcastle W. Inhibiting an abnormal lingual pattern in a cleft palate child using electropalatography. In: Leahy MM, Kallen JL editor(s). Interdisciplinary perspectives in speech and language pathology. Dublin: School of Clinical Speech and Language Studies, 1992:211-221.

\section{Fujiwara 2007 \{published data only\}}

Fujiwara Y. Electropalatography home training using a portable training unit for Japanese children with cleft palate. Advances in SpeechLanguage Pathology 2007;9(1):65-72.

\section{Gibbon 1989 \{published data only\}}

Gibbon F, Hardcastle W. Deviant articulation in a cleft palate child following late repair of the hard palate: A description and remediation procedure using electropalatography (EPG). Clinican Linguistics \& Phonetics 1989;3(1):93-110.

\section{Gibbon 1998 \{published data only\}}

Gibbon F, Crampin L, Hardcastle B, Nairn M, Razzell R, Harvey L, Reynolds B. CLEFTNET (SCOTLAND): A network for the treatment of cleft palate speech using EPG. International Journal of Language and Communication Disorders 1998;33(suppl.):44-49.

Gibbon 2001 \{published data only\}

Gibbon FE, Hardcastle WJ, Crampin L, Reynolds B, Razzell R, Wilson J. Visual feedback therapy using electropalatography (EPG) for articulation disorders associated with cleft palate. Asia Pacific Journal of Speech, Language and Hearing 2001;6:53-58.

Hardcastle 1989 \{published data only\}

Hardcastle W, Morgan Barry R, Nunn M. Instrumental articulatory phonetics in assessment and remediation: Case studies with the electropalatograph. In: Stengelhofen J editor(s). Cleft palate: The nature and remediation of communicative problems. Edinburgh: Churchill Livingstone, 1989:136-164

Michi 1985 \{published data only\}

Michi K, Yamashita Y, Suzuki N, Imai S. Use of the multi-function speech training aid in the treatment of articulation disorders: Serial observation of a case of cleft palate. Proceedings of the 6th International Congress on Cleft Palate and Related Craniofacial Anomalies. Monte Carlo, Monaco, 2nd-7th September, 1985:111.

\section{Michi 1986 \{published data only\}}

Michi K, Suzuki N, Yamshiata Y, Imai S. Visual training and correction of articulation disorders by ise of dynamic palatography: Serial observation in a case of cleft palate. Journal of Speech and Hearing Disorders 1986;51:226-238.

Michi 1990 \{published data only\}

Michi K, Yamashita Y, Imai S, Ohno K. Results of treatment of speech disorders in cleft palate patients: Patients obtaining adequate velopharyngeal function. In: Pfeifer G editor(s). Craniofacial abnormalities and clefts of the lip, alveolus and palate. Stuttgart: Thieme, 1990:419-423.

Ohira 1989 \{published data only\}

Ohira A. Visual feedback training for velopharyngeal closure by using the speech aid appliance equipped with a bulb with electrodes. Journal of Japanese Cleft Palate Association 1989;14:412-420.

Schmidt 2007 \{published data only\}

Schmidt AM. Evaluating a new clinical palatometry system. Advances in Speech-Language Pathology 2007;9(1):73-81.

Scobbie 2004 \{published data only\}

Scobbie JM, Wood SE, Wrench AA. Advances in EPG for treatment and research: An illustrative case study. Clinical Linguistics \& Phonetics 2004;18(6-8):373-389.

Stokes 1996 \{published data only\}

Stokes SF, Whitehill TL, Yuen KCP, Tsui AMY. EPG treatment of sibilants in two Cantonese-speaking children with cleft palate. Clinical Linguistics \& Phonetics 1996;10(4):265-280.

Whitehill 1996 \{published data only\}

Whitehill TL, Stokes SF, Man YHY. Electropalatography treatment in an adult with late repair of cleft palate. Cleft Palate-Craniofacial Journal 1996;33(2):160-168.

Yamashita 1988 \{published data only\}

Yamashita Y, Michi K. The efficacy of dynamic palatography for the treatment of articulation disorders in cleft palate patients: The assessment of the therapeutic process for the /s/ sound. Journal of Japanese Cleft Palate Association 1988;13:242-252.

Yamashita 1999 \{published data only\}

Yamashita Y, Suzuki N, Imai S, Mori K, Takahashi K, Michi K. The efficacy of the speech training system for the treatment of articulation disorders in patients with cleft palate. Journal of Japanese Cleft Palate Association 1999;24(1):52-60.

\section{Additional references}

\section{Anthony 1971}

Anthony A, Bogle D, Ingram TTS, McIsaac MW. EAT: The Edinburgh Articulation Test. Edinburgh: Livingstone, 1971.

\section{CLAPA 2001}

Cleft Lip and Palate Association. What is cleft lip \& palate?. CLAPA website 2001.

CSAG 1998

Clinical Standards Advisory Group. Clinical effectiveness: Report of the Clinical Standards Advisory Group. London: HMSO, 1998.

\section{Dalston 1992}

Dalston RM. Timing of cleft palate repair: A speech pathologist's viewpoint. Problems in Plastic and Reconstructive Surgery 1992;2:30 38.

\section{Davis 1980}

Davis SM, Drichta CE. Biofeedback theory and application in allied health. Biofeedback and Self-Regulation 1980;5(2):159-174.

Donner 2000

Donner A, Klar N. Design and analysis of cluster randomization trials in health research. London: Arnold, 2000. 


\section{Donner 2001}

Donner A, Piaggio G, Villar J. Statistical methods for the metaanalysis of cluster randomization trials. Statistical Methods in Medical Research 2001;10:325-338.

\section{Egger 1997}

Egger M, Davey Smith G, Schneider M, Minder C. Bias in metaanalysis detected by a simple, graphic test. BMJ 1997;315(7):629634.

\section{Endriga 1999}

Endriga MC, Kapp-Simon KA. Psychological issues in craniofacial care: State of the art. Cleft Palate-Craniofacial Journal 1999;36(1): $3-11$.

\section{Fairbanks 1954}

Fairbanks G. Systematic research in experimental phonetics: 1. A theory of the speech mechanism as a servosystem. Journal of Speech and Hearing Disorders 1954;19:133-139.

\section{Gibbon 2006}

Gibbon FE, Patterson L. A survey of speech and language therapists' views on electropalatography therapy outcomes in Scotland. Child Language Teaching and Therapy 2006;22(3):275-292.

\section{Gibbon 2007}

Gibbon FE. Bibliography of electropalatography (EPG) studies in English (1957-2007). Queen Margaret University website 2007.

\section{Hardcastle 1991}

Hardcastle WJ, Gibbon FE, Jones W. Visual display of tongue-palate contact: Electropalatography in the assessment and remediation of speech disorders. British Journal of Disorders of Communication 1991; 26:41-74.

\section{Hardcastle 1997}

Hardcastle WJ, Gibbon FE. Electropalatography and its clinical applications. In: Ball MJ, Code C editor(s). Instrumental Clinical Phonetics. London: Whurr Publisher, 1997:149-193.

\section{Hardin-Jones 2005}

Hardin-Jones MA, Jones DL. Speech production of preschoolers with cleft palate. Cleft Palate-Craniofacial Journal 2005;42(1):7-13.

\section{Higgins 2002}

Higgins JPT, Thompson SG. Quantifying heterogeneity in a metaanalysis. Statistics in Medicine 2002;21:1539-1558.

\section{Higgins 2003}

Higgins JPT, Thompson SG, Deeks JJ, Altman DG. Measuring inconsistency in meta-analyses. BMJ 2003;327:557-560.

\section{Higgins 2005}

Higgins JPT, Green S, editors. Cochrane Handbook for Systematic Reviews of Interventions 4.2.5 [updated May 2005]. The Cochrane Library, Issue 3, 2005. Chichester, UK: John Wiley \& Sons, Ltd, 2005.

\section{Higgins 2008}

Higgins JPT, Green S, editors. Cochrane Handbook for Systematic Reviews of Interventions Version 5.0.1 [updated September 2008]. The Cochrane Collaboration, 2008. Available from www.cochranehandbook.org.

\section{Juni 2001}

Juni P, Altman DG, Egger M. Assessing the quality of randomised controlled trials. In: Egger M, Smith GD, Altman DG editor(s).
Systematic reviews in health care: Meta-analysis in context. London: BMJ Books, 2001:87-108.

\section{Kummer 2001}

Kummer AW. Cleft palate and craniofacial anomalies: The effects on speech and resonance. Clifton Park, NY: Thomson Delmar Learning, 2008.

Lee 2007

Lee A, Gibbon FE, Crampin L, Yuen I, McLennan G. The national CLEFTNET project for individuals with speech disorders associated with cleft palate. Advances in Speech-Language Pathology 2007;9(1): 57-64

\section{Liao 2006}

Liao Y-F, Mars M. Hard palate repair timing and facial growth in cleft lip and palate: A systematic review. Cleft Palate-Craniofacial Journal 2006;43(5):563-570.

\section{Marazita 2004}

Marazita ML, Mooney MP. Current concepts in the embryology and genetics of cleft lip and cleft palate. Clinics in Plastic Surgery 2004; 31:125-140

\section{Michi 1986}

Michi K, Suzuki N, Yamshiata Y, Imai S. Visual training and correction of articulation disorders by ise of dynamic palatography: Serial observation in a case of cleft palate. Journal of Speech and Hearing Disorders 1986;51:226-238.

\section{Michi 1990}

Michi K, Yamashita Y, Imai S, Ohno K. Results of treatment of speech disorders in cleft palate patients: Patients obtaining adequate velopharyngeal function. In: Pfeifer G editor(s). Craniofacialabnormalities and clefts of the lip, alveolus and palate. Stuttgart: Thieme, 1990:419-423.

MRC 2000

Medical Research Council. A framework for development and evaluation of RCTs for complex interventions to improve health. London, England: Medical Research Council, 2000.

Mysak 1959

Mysak Ed. A servo model for speech therapy. Journal of Speech and Hearing Disorders 1959;24:144-149.

\section{NICE 2002}

National Institute for Clinical Excellence. Interventional procedures overview of electropalatography. Interventional procedures programme 2002.

\section{Nopoulos 2002}

Nopoulos P, Berg S, Canady J, Richman L, Van Demark D, Andreasen NC. Structural brain abnormalities in adult males with clefts of the lip and/or palate. Genetics in Medicine 20002;4(1):1-9.

\section{Paliobei 2005}

Paliobei V, Psifidis A, Anagnostopoulos D. Hearing and speech assessment of cleft palate patients after palatal closure: Long-terms results. International Journal of Pediatric Otorhinolaryngology 2005;69: 1373-1381.

\section{Peterson 1990}

Peterson-Falzone SJ. A cross-sectional analysis of speech results following palatal closure. In: Bardach J, Morris HL editor(s). Multidisciplinary management of cleft lip and palate. Philadelphia: W B Saunders, 1990:750-756. 


\section{Peterson 2001}

Peterson-Falzone SJ, Hardin-Jones MA, Karnell MP. Cleft palate speech. Third Edition. St. Louis: Mosby, 2001.

\section{Peterson 2006}

Peterson-Falzone SJ, Trost-Cardamone JE, Karnell MP, Hardin-Jones MA. The clinician's guide to treating cleft palate speech. St. Louis, MO: Mosby, 2006.

\section{RCSLT 2005}

Royal College of Speech and Language Therapists. Royal College of Speech and Language Therapists Clinical Guidelines. Oxon, UK Speechmark Publishing Ltd., 2005.

\section{Robey 1998}

Robey RR, Schultz MC. A model for conducting clinical-outcome research: An adaptation of the standard protocol for use in aphasiology. Aphasiology 1998;12(9):787-810.

\section{Seagle 2004}

Seagle MB. Primary surgical correction of cleft lip and palate. In: Bzoch KR editor(s). Communicative disorders related to cleft lip and palate. Fifth. Austin, TX: Pro-ed, 2004:181-192.

\section{Sell 2001}

Sell DA, Grunwell P. Speech assessment and therapy. In: Watson ACH, Sell DA, Grunwell P editor(s). Management of cleft lip and palate. London: Whurr Publishers, 2001:227-257.

\section{Sellars 2005}

Sellars C, Hughes T, Langhorne P. Speech and language therapy for dysarthria due to non-progressive brain damage (Cochrane Review). Cochrane Database of Systematic Reviews 2005, Issue 3. [DOI: 10.1002/14651858.CD002088.pub2]

\section{Smith 1967}

Smith KU, Henry JP. Cybernetic foundations for rehabilitation. American Journal of Physical Medicine 1967;46:379-467.

\section{Stengelhofen 1990}

Stengelhofen J. Working with cleft palate. Bicester: Winslow Press, 1990.

\section{Warren 1990}

Warren DW, Dalston RM, Dalston ET. Maintaining speech pressures in the presence of velopharyngeal impairment. Cleft Palate Journal 1990;27(1):53-60.

\section{Watson 2001}

Watson 2001. Primary surgery. In: Watson ACH, Sell DA, Grunwell P editor(s). Management of cleft lip and palate. London: Whurr Publishers, 2001:157-183.

\section{Wiener 1948}

Wiener N. Cybernetics. New York: Wiley, 1948.

\section{Winter 2000}

Winter R, Baraitser M. London dysmorphology database \& London neurogenetics database [CD-ROM]. Oxford: Oxford University Press, 2000.

\section{Witzel 1991}

Witzel MA. Speech evaluation and treatment. Oral and Maxillofacial Surgery Clinics of North America 1991;3:501-516.

\section{Wrench 2007}

Wrench AA. Advances in EPG palate design. Advances in SpeechLanguage Pathology 2007;9(1):3-12.

\section{Yamashita 1992}

Yamashita Y, Michi K, Imai S, Suzuki N, Yoshida H. Electropalatographic investigation of abnormal lingual-palatal contact patterns in cleft palate patients. Clinical Linguistics \& Phonetics 1992;6(3):201217.

* Indicates the major publication for the study 
CHARACTERISTICS OF STUDIES

Characteristics of included studies [ordered by study ID]

Michi 1993

\begin{tabular}{ll}
\hline Methods & Randomised controlled trial using parallel group design \\
\hline Participants & $\begin{array}{l}6 \text { participants ( } 4 \text { males and } 2 \text { females) aged } 4-6 \text { years. } 3 \text { of them had unilateral cleft lip } \\
\text { and palate and } 3 \text { had bilateral cleft lip and palate. All showed compensatory articulation } \\
\text { errors - palatalized } / \mathrm{s} / .\end{array}$ \\
\hline Interventions & $\begin{array}{l}\text { Participants received a } 60 \text {-minute individual therapy session once per week, except for } \\
1 \text { who had daily treatment: } \\
2 \text { participants received EPG therapy and frication display method; } \\
2 \text { received EPG therapy; } \\
2 \text { received no-visual-feedback. } \\
\text { Therapy targeted fricative } / \mathrm{s} / .\end{array}$ \\
\hline
\end{tabular}

Outcomes

The outcome measures were percent correction production of tongue placement, determined on the basis of EPG contact pattern, and frication, as by perceptual evaluation by the second author. The data was collected at the start and end of each session. The treatment at a certain level continued and did not progress to the next until the participants achieved at least $80 \%$ accuracy. The number of therapy sessions required to attain each stage for each participants was selectively reported in the Results section.

\section{Notes}

\section{Risk of bias}

\begin{tabular}{|c|c|c|}
\hline Item & Authors' judgement & Description \\
\hline Adequate sequence generation? & Unclear & $\begin{array}{l}\text { The authors stated that "each subject was randomly assigned } \\
\text { and treated by one of the following three methods..." (P. 279). } \\
\text { Randomisation was probably done but the method used for } \\
\text { generating the randomised sequence was not described. }\end{array}$ \\
\hline Allocation concealment? & No & $\begin{array}{l}\text { The authors stated that "treatment was carried out by the same } \\
\text { speech-language pathologist" (P. 279). Allocation was probably } \\
\text { not concealed from the healthcare provider. }\end{array}$ \\
\hline $\begin{array}{l}\text { Blinding? } \\
\text { All outcomes }\end{array}$ & No & $\begin{array}{l}\text { Frication production was assessed in real time by the second au- } \\
\text { thor. The speech samples were recorded and played back at ran- } \\
\text { dom to the same author for assessing interjudge agreement; } 90 \% \\
\text { consistency was reported. The authors did not state whether the }\end{array}$ \\
\hline
\end{tabular}


Continued)

\begin{tabular}{|c|c|c|}
\hline & & $\begin{array}{l}\text { second author was blinded to treatment allocation at the time } \\
\text { of assessment. }\end{array}$ \\
\hline $\begin{array}{l}\text { Incomplete outcome data addressed? } \\
\text { All outcomes }\end{array}$ & Yes & No drop-out was reported. \\
\hline Free of selective reporting? & No & $\begin{array}{l}\text { The authors mentioned measuring percent correct production } \\
\text { for tongue placement and frication for assessing the treatment } \\
\text { progress in the Method section, however, they focused on re- } \\
\text { porting the number of therapy sessions each participant required } \\
\text { to achieve the target for some of the stages of the interventions. } \\
\text { Charts were included to provide additional information on this } \\
\text { but they were difficult to interpret. }\end{array}$ \\
\hline Free of other bias? & Yes & $\begin{array}{l}\text { All participants showed the same type of articulation errors be- } \\
\text { fore the intervention began. No early stopping of intervention } \\
\text { and co-intervention were reported. }\end{array}$ \\
\hline
\end{tabular}

Characteristics of excluded studies [ordered by study ID]

\begin{tabular}{ll}
\hline Dent 1992 & Not RCT (case study) \\
\hline Fujiwara 2007 & Not RCT (case study series) \\
\hline Gibbon 1989 & Not RCT (case study) \\
\hline Gibbon 1998 & Not RCT (case study) \\
\hline Gibbon 2001 & Cross-over trial design, which is inappropriate for evaluating speech intervention, was used. \\
\hline Hardcastle 1989 & Not RCT (case study) \\
\hline Michi 1985 & Not RCT (case study) \\
\hline Michi 1986 & Not RCT (case study) \\
\hline Michi 1990 & Not RCT (before and after study, without control group) \\
\hline Ohira 1989 & Not RCT (case study) \\
\hline Schmidt 2007 & Not RCT (case study series)
\end{tabular}




\section{(Continued)}

\begin{tabular}{ll} 
Scobbie 2004 & Not RCT (case study) \\
\hline Stokes 1996 & Not RCT (case study series) \\
\hline Whitehill 1996 & Not RCT (case study) \\
\hline Yamashita 1988 & Not RCT (case study series) \\
\hline Yamashita 1999 & Not RCT (before and after study, without control group) \\
\hline
\end{tabular}




\section{DATA AND ANALYSES}

This review has no analyses.

\section{A P PENDICES}

\section{Appendix I. CENTRAL search strategy}

CENTRAL searched via the Cochrane Library Issue 1, 2008

1. cleft palate

2. electropalatograph*

3. EPG

4. palatograph*

5. palatomet*

6. 2 or 3 or 4 or 5

7. 1 and 6

\section{Appendix 2. MEDLINE search strategy}

MEDLINE searched via OCLC 1966 to March 2008

1. cleft

2. palate

3. electropalatograph*

4. EPG

5. palatograph*

6. palatomet*

7. 1 and 2

8. 3 or 4 or 5 or 6

9.7 and 8

\section{Appendix 3. EMBASE search strategy}

EMBASE searched 1974 to March 2008

1. cleft

2. palate

3. electropalatograph*

4. EPG

5. palatograph*

6. palatomet*

7. 1 and 2

8.3 or 4 or 5 or 6

9.7 and 8 


\section{Appendix 4. ERIC search strategy}

ERIC searched via OCLC 1966 to March 2008

1. cleft

2. palate

3. electropalatograph*

4. EPG

5. palatograph*

6. palatomet*

7. 1 and 2

8. 3 or 4 or 5 or 6

9.7 and 8

\section{Appendix 5. PsyclNFO search strategy}

PsycINFO searched via CSA 1967 to March 2008

1. cleft palate

2. electropalatograph*

3. EPG

4. palatograph*

5. palatomet*

6. 2 or 3 or 4 or 5

7. 1 and 6

\section{Appendix 6. CINAHL search strategy}

CINAHL searched via Ovid 1982 to March 2008

1. cleft palate

2. electropalatograph*

3. EPG

4. palatograph*

5. palatomet*

6. 2 or 3 or 4 or 5

7. 1 and 6

Appendix 7. Linguistics and Language Behaviour Abstracts search strategy

LLBA searched via CSA 1973 to March 2008

1. cleft palate

2. electropalatograph*

3. EPG

4. palatograph*

5. palatomet*

6. 2 or 3 or 4 or 5

7. 1 and 6 


\section{Appendix 8. Allied and Complementary Medicine search strategy}

AMED searched via Ovid 1985 to March 2008

Terms used:

cleft palate

\section{Appendix 9. LILACS search strategy}

LILACS searched via Virtual Health Library 1982 to March 2008

Terms used:

cleft palate AND electropalatograph* or EPG or palatograph* or palatomet*

\section{Appendix 10. National Research Register}

The National Research Register searched March 2008

Terms used:

electropalatography or EPG or palatometer or palatography

\section{Appendix II. ClinicalTrials.gov search strategy}

ClinicalTrials.gov searched March 2008

Terms used:

electropalatography

\section{Appendix 12. Dissertation Abstracts search strategy}

Dissertation Abstracts searched via Digital dissertation consortium 1861 to March 2008 Terms used:

cleft palate AND electropalatography

\section{WHAT'S NEW}

Last assessed as up-to-date: 8 April 2008.

9 May 2009 Amended Post-hoc change in the protocol - Unit of analysis issues added; cross-over trials considered as inappropriate for speech intervention. 


\section{H I S T O R Y}

Protocol first published: Issue 4, 2007

Review first published: Issue 3, 2009

\section{CONTRIBUTIONSOFAUTHORS}

FG and AL conducted this review; the searches were run by AL, JL was available for independent assessment of any trials identified, where necessary. All reviewers contributed to the writing of the protocol and the review.

\section{DECLARATIONS OF INTEREST}

FG has written extensively on this topic.

\section{DIFFERENCES BETWEEN PROTOCOL AND REVIEW}

Post-hoc change in the protocol - a section under 'Unit of analysis' issues was added and a decision taken that cross-over trials were considered as inappropriate for speech intervention and would therefore be excluded from this version of the review and from future updates. 